\title{
More iOS6 Development
} Further Explorations of the iOS SDK

\author{
Alex Horovitz \\ Kevin Kim \\ Jeff LaMarche \\ David Mark
}




\title{
More iOS6 Development
}

\author{
Copyright $\odot 2013$ by Alex Horovitz, Kevin Kim, Jeff LaMarche and David Mark
}

This work is subject to copyright. All rights are reserved by the Publisher, whether the whole or part of the material is concerned, specifically the rights of translation, reprinting, reuse of illustrations, recitation, broadcasting, reproduction on microfilms or in any other physical way, and transmission or information storage and retrieval, electronic adaptation, computer software, or by similar or dissimilar methodology now known or hereafter developed. Exempted from this legal reservation are brief excerpts in connection with reviews or scholarly analysis or material supplied specifically for the purpose of being entered and executed on a computer system, for exclusive use by the purchaser of the work. Duplication of this publication or parts thereof is permitted only under the provisions of the Copyright Law of the Publisher's location, in its current version, and permission for use must always be obtained from Springer. Permissions for use may be obtained through RightsLink at the Copyright Clearance Center. Violations are liable to prosecution under the respective Copyright Law.

ISBN 978-1-4302-3807-2

ISBN 978-1-4302-3808-9 (eBook)

Trademarked names, logos, and images may appear in this book. Rather than use a trademark symbol with every occurrence of a trademarked name, logo, or image we use the names, logos, and images only in an editorial fashion and to the benefit of the trademark owner, with no intention of infringement of the trademark.

The use in this publication of trade names, trademarks, service marks, and similar terms, even if they are not identified as such, is not to be taken as an expression of opinion as to whether or not they are subject to proprietary rights.

While the advice and information in this book are believed to be true and accurate at the date of publication, neither the authors nor the editors nor the publisher can accept any legal responsibility for any errors or omissions that may be made. The publisher makes no warranty, express or implied, with respect to the material contained herein.

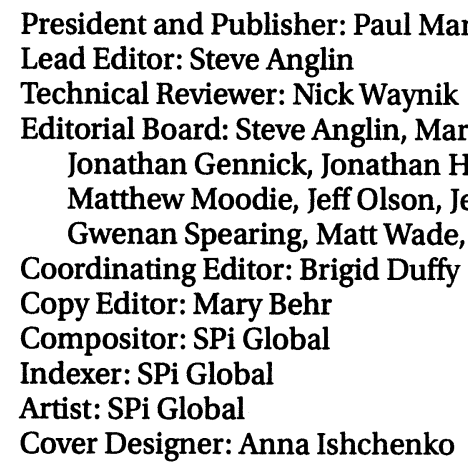

Distributed to the book trade worldwide by Springer Science+Business Media New York, 233 Spring Street, 6th Floor, New York, NY 10013. Phone 1-800-SPRINGER, fax (201) 348-4505, e-mail orders-ny@springer-sbm. com, or visit WWW. springeronline. com. Apress Media, LLC is a California LLC and the sole member (owner) is Springer Science + Business Media Finance Inc (SSBM Finance Inc). SSBM Finance Inc is a Delaware corporation.

For information on translations, please e-mail rights@apress .com, or visit www. apress .com.

Apress and friends of ED books may be purchased in bulk for academic, corporate, or promotional use. eBook versions and licenses are also available for most titles. For more information, reference our Special Bulk Sales-eBook Licensing web page at www. apress. com/bulk-sales.

Any source code or other supplementary materials referenced by the author in this text is available to readers at www. apress. com. For detailed information about how to locate your book's source code, go to www. apress. com/ source-code. 
To Annie, for all her love and support. 


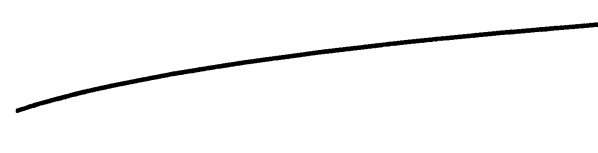

\section{Contents at a Glance}

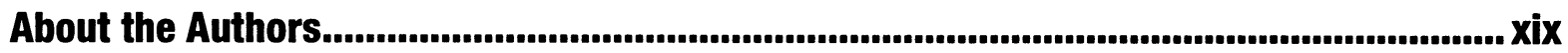
About the Technical Reviewer .................................................................................................... xxi Acknowledgments ............................................................................................................................. xxiii

Chapter 1: Here We Go Round Again.....................................................................................1

Chapter 2: Core Data: What, Why, and How .........................................................................7

Chapter 3: A Super Start: Adding, Displaying, and Deleting Data.........................................39

Chapter 4: The Devil in the Detail View ..................................................................................87

Chapter 5: Preparing for Change: Migrations and Versioning...........................................123

Chapter 6: Custom Managed Objects .........................................................................................133

Chapter 7: Relationships, Fetched Properties and Expressions .......................................173

Chapter 8: Behind Every iCloud ................................................................................................223

Chapter 9: Peer-to-Peer Over Bluetooth Using Game Kit...................................................251

Chapter 10: Map Kit......................................................................................................................295

Chapter 11: Messaging: Mail, SMS, and Social Media ................................................323

Chapter 12: Media Library Access and Playback ...........................................................337

Chapter 13: Locking It Down: iOS Security.......................................................................405 
Chapter 14: Keeping Your Interface Responsive .........................................................443

Chapter 15: Unit Testing, Debugging, and Instruments ...........................................481

Chapter 16: The Road Goes Ever On... ..................................................................511

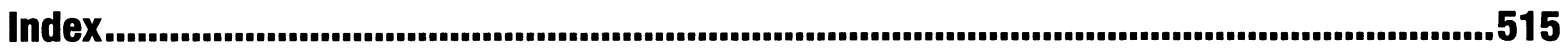




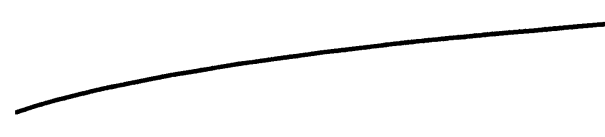

\section{Contents}

About the Authors.......................................................................................................... xix

About the Technical Reviewer ........................................................................................ xxi

Acknowledgments ................................................................................................................ . xxiii

Chapter 1: Here We Go Round Again...............................................................................1

What This Book Is................................................................................................................

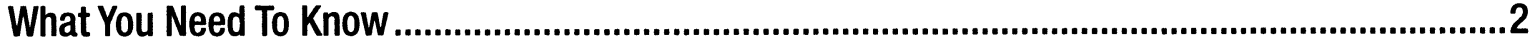

What You Need Before You Can Begin .........................................................................................2

What's In this Book ...............................................................................................................

Chapter 2: Core Data: What, Why, and How ........................................................................7

A Brief History of Core Data..........................................................................................................................

Creating a Core Data Application .................................................................................................................

Core Data Concepts and Terminology ..................................................................................................12

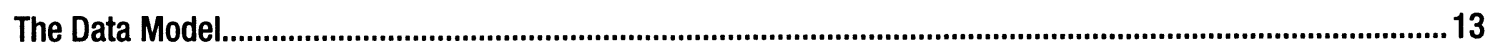

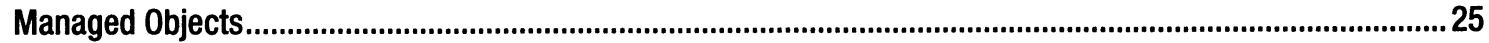

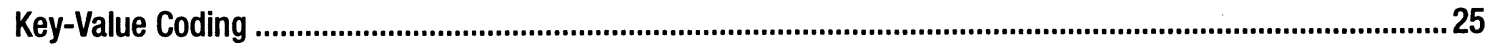

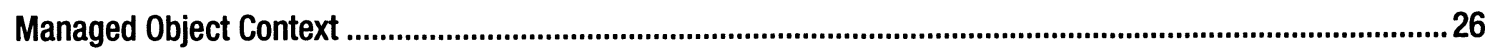

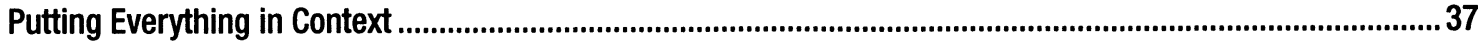


Chapter 3: A Super Start: Adding, Displaying, and Deleting Data 39

Setting up the Xcode Project

Adding a Scene. 48

Scenes and Segues 48

Storyboard Document Outline 49

Application Architecture .50

Designing the View Controller Interface .51

Creating HeroListController .56

Making the Connections and Outlets 60

Navigation Bar Buttons. .63

Tab Bar and User Defaults 66

Designing the Data Model .67

Adding an Entity 68

Editing the New Entity 68

Adding Attributes to the Hero Entity .70

Declaring the Fetched Results Controller .76 Implementing the Fetched Results Controller ..........................................................................77

Fetched Results Controller Delegate Methods .................................................................................79

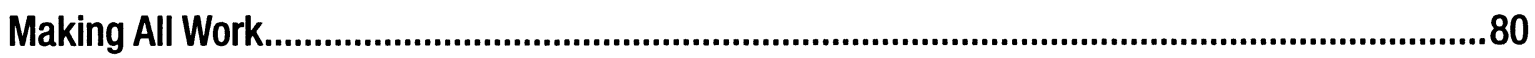

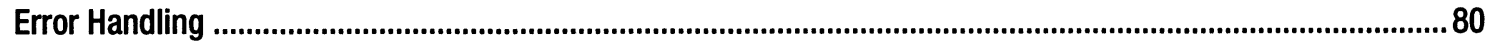

Implementing Edit and Add ......................................................................................................................

Coding the Table View Data Source and Delegate ........................................................................................ 82

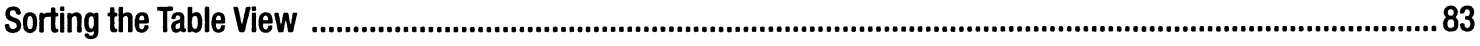

Loading the Fetch Request At Launch ............................................................................................................ 84

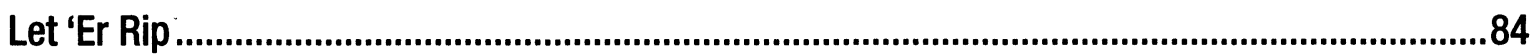

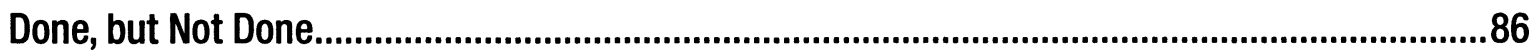

Chapter 4: The Devil in the Detail View .........................................................................87

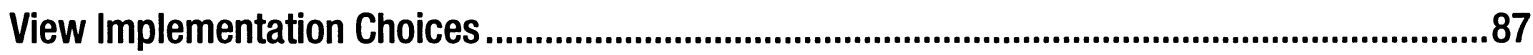

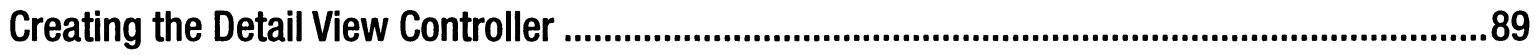

Wiring Up the Segue ...................................................................................................................94

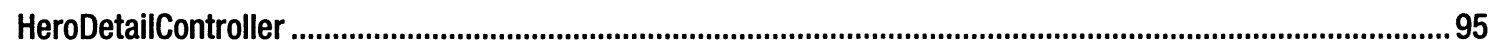




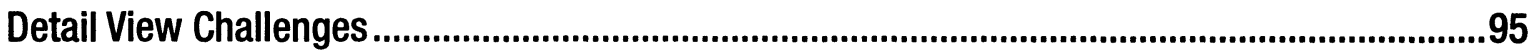

Controlling the Table Structure with Property Lists................................................................97

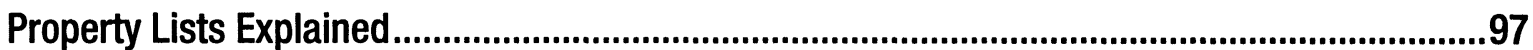

Modeling Table Structure with a Property List...................................................................................................98

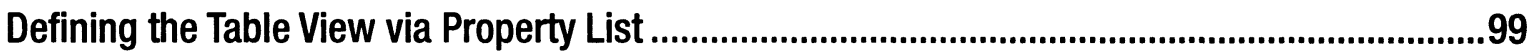

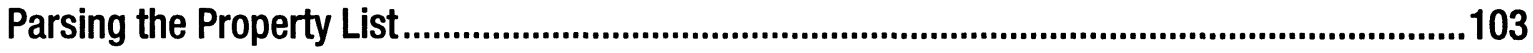

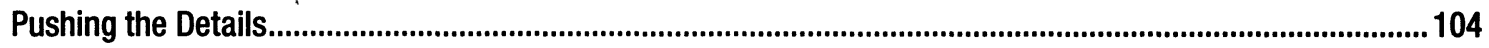

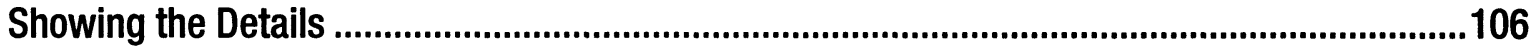

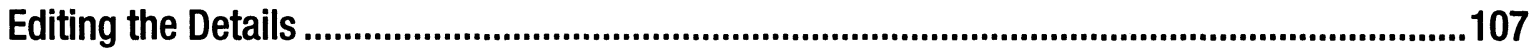

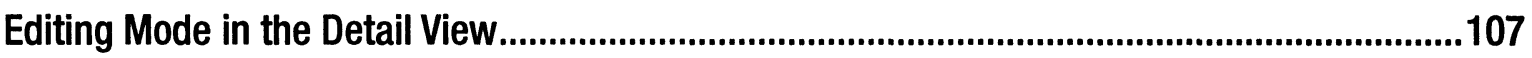

Creating a Custom UITableViewCell Subclass .........................................................................110

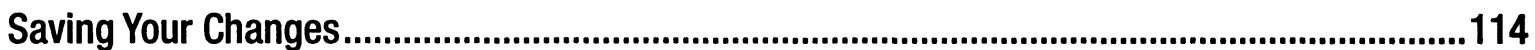

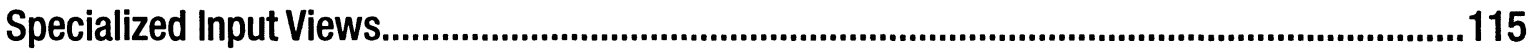

DatePicker SuperDBEditCell Subclass..........................................................................................................115

Using the DatePicker SuperDBEditCell Subclass..........................................................................................117

Implementing a Selection Picker..........................................................................................................119

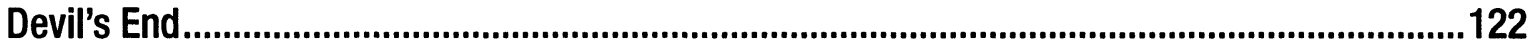

Chapter 5: Preparing for Change: Migrations and Versioning .........................................123

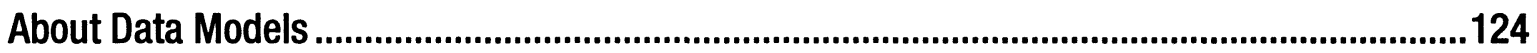

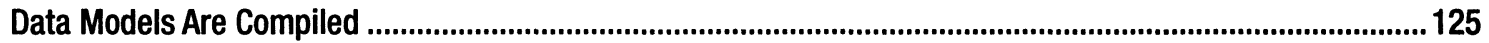

Data Models Can Have Multiple Versions ....................................................................................................... 126

Creating a New Data Model Version ..........................................................................................................126

The Current Data Model Version ........................................................................................................................128

Data Model Version Identifiers...................................................................................................................... 128

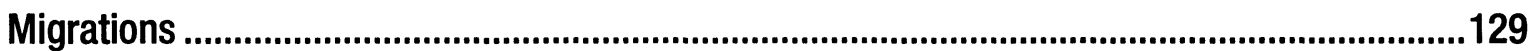

Lightweight vs. Standard.................................................................................................................. 130

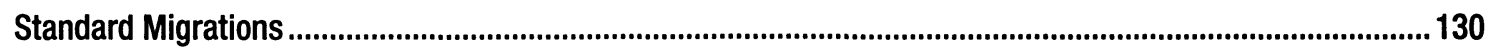

Setting Up Your App to Use Lightweight Migrations .....................................................................130

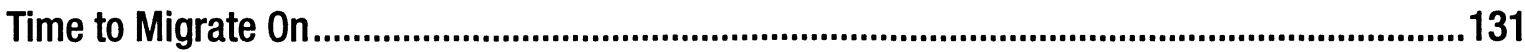


Chapter 6: Custom Managed Objects 133

Updating the Data Model 137

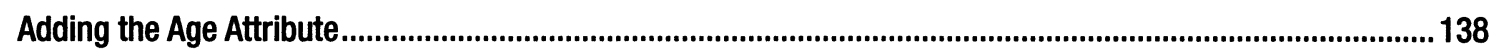

Adding the Favorite Color Attribute.....................................................................................................138

Adding a Minimum Length to the Name Attribute .................................................................................. 139

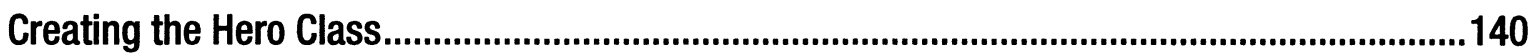

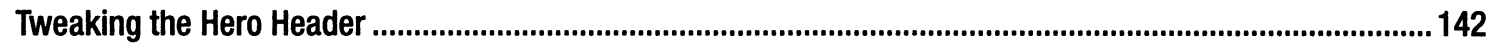

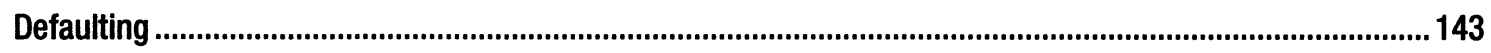

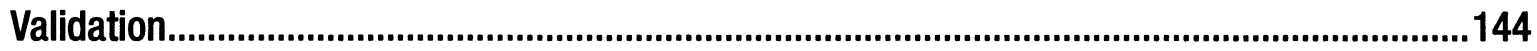

Single-Attribute Validations ................................................................................................................ 145

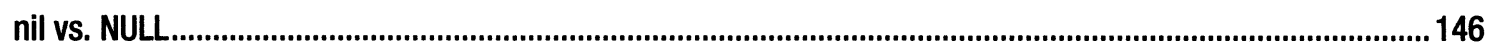

Multiple-Attribute Validations ..............................................................................................................147

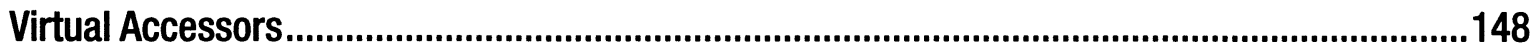

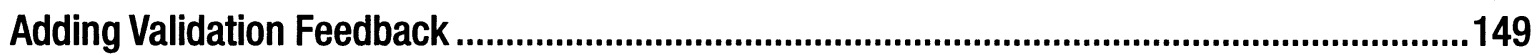

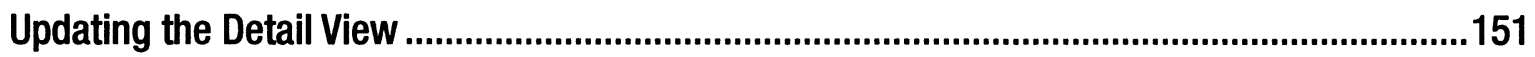

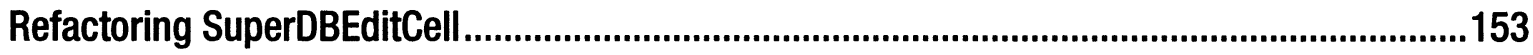

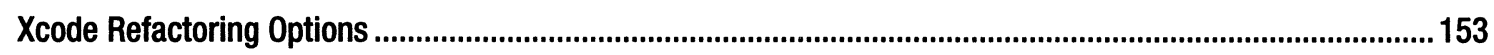

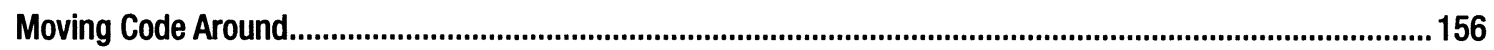

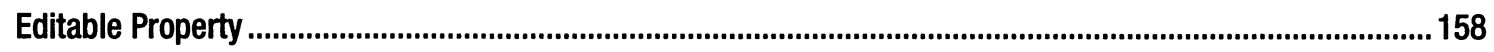

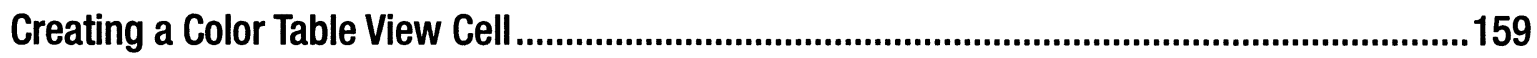

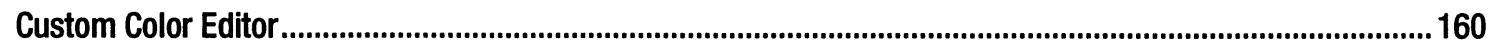

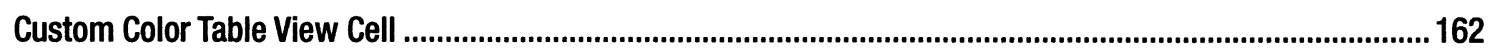

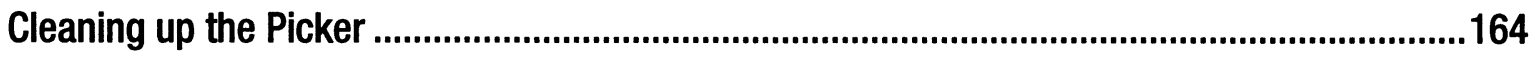

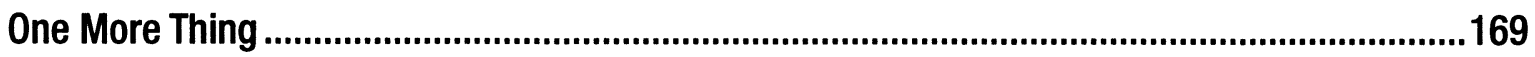

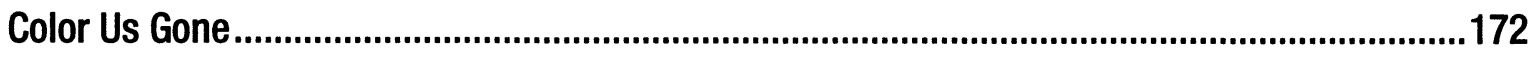

Chapter 7: Relationships, Fetched Properties and Expressions ......................................173

Expanding Your Application: Superpowers and Reports.......................................................173

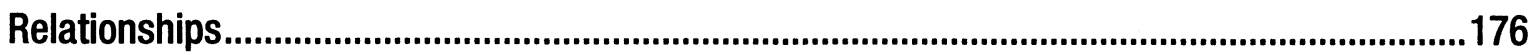

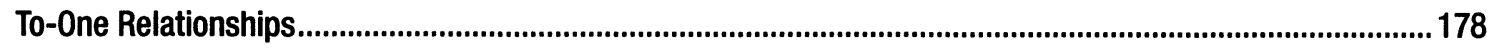

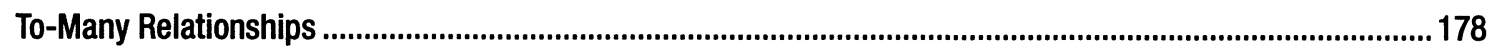


Inverse Relationships 180

Fetched Properties 181

Creating Relationships and Fetched Properties in the Data Model Editor 182 Delete Rules

Expressions and Aggregates 183

Adding the Power Entity.

Creating the Powers Relationship .................................................................................186

Creating the Inverse Relationship .................................................................................188

Creating the olderHeroes Fetched Property ......................................................................188

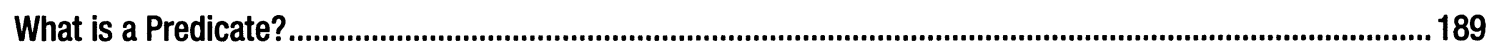

Creating the youngerHeroes Fetched Property .......................................................................190

Creating the sameSexHeroes Fetched Property.....................................................................191

Creating the oppositeSexHeroes Fetched Property.................................................................191

Adding Relationships and Fetched Properties to the Hero Class .............................................191

Updating the Detail View ......................................................................................................192

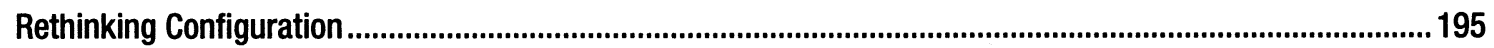

Encapsulation and Information Hiding................................................................................................... 199

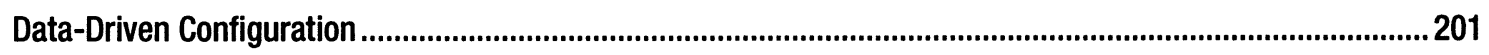

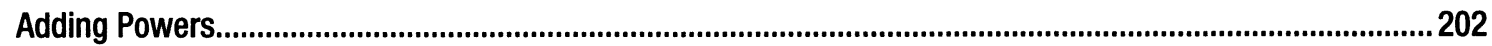

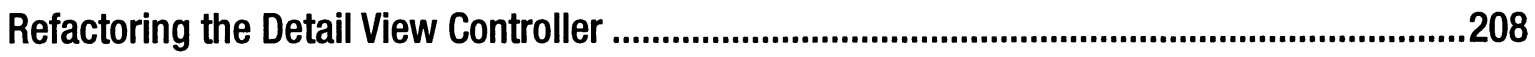

Renaming the Configuration Class ..........................................................................................................208

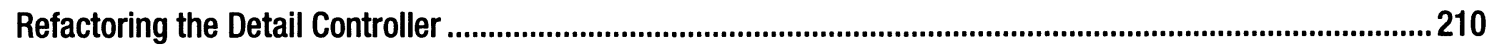

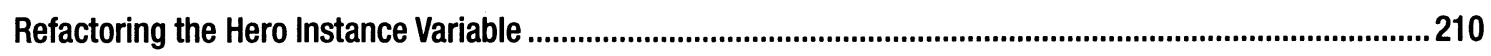

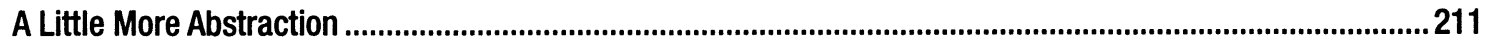

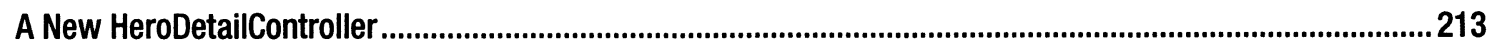

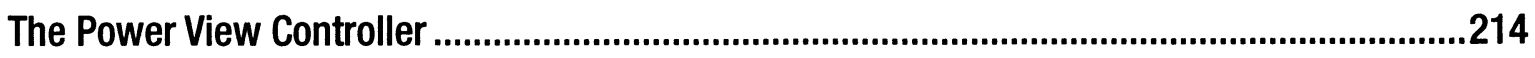

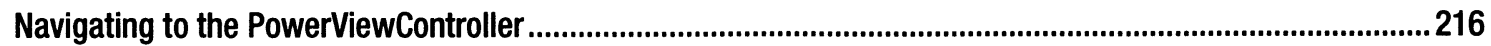

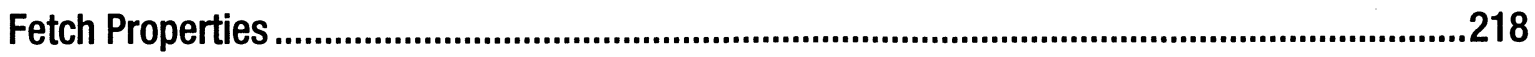

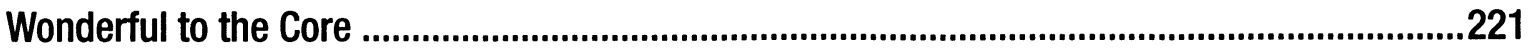


Chapter 8: Behind Every iCloud 223

Data Storage with iCloud. .223

iCloud Basics. 224

iCloud Backup 224

Enabling iCloud in Your Application 225 Key-Value Data Storage .225

Document Storage 226

UIDocument. .226

UIDocument with iCloud 230

NSMetadataQuery. 231

Core Data with iCloud. 233

Enhancing SuperDB 234

Entitlements 235

Creating an iCloud enabled Provisioning Profile. 236

Updating the Persistent Store.. 245

Updating the Managed Object Context. 247

Updating the UI on DataChanged. 248

Testing the Data Store . 249

Keep Your Feet on the Ground.

\section{Chapter 9: Peer-to-Peer Over Bluetooth Using Game Kit}

Game Center

Peer-to-Peer Connectivity

In Game Voice.

This Chapter's Application

Network Communication Models

Client-server Model

Peer-to-Peer Model

Hybrid Client-server/Peer-to-Peer 
The Game Kit Session

Creating the Session............................................................................................................................ 265

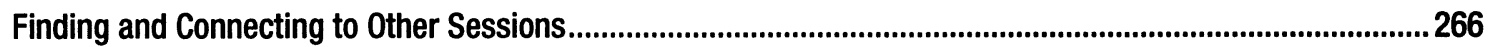

Listening for Other Sessions............................................................................................................................ 266

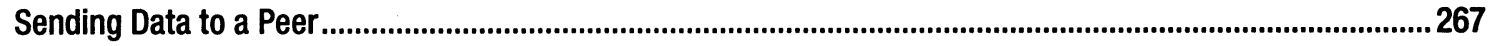

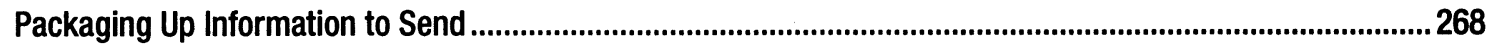

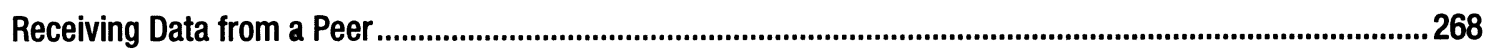

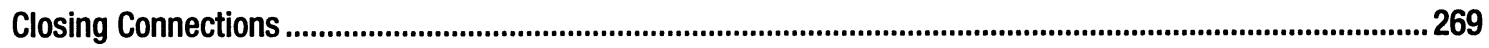

The Peer Picker

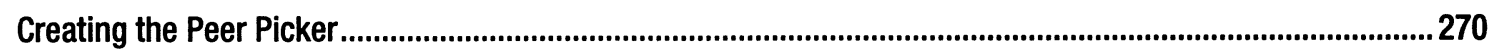

Handling a Peer Connection ......................................................................................................................... 270

Creating the Session..................................................................................................................................... 270

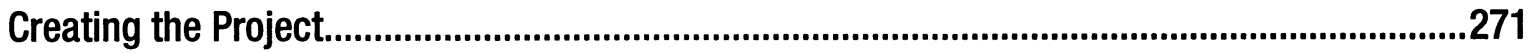

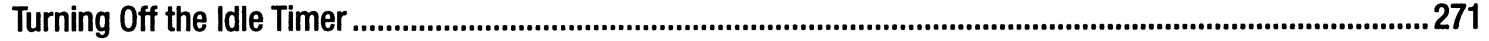

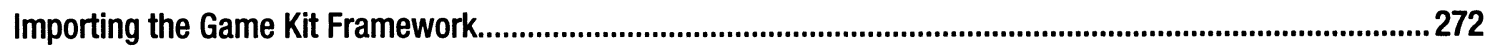

Designing the Interface ........................................................................................................................... 272

Defining Application Constants.............................................................................................................. 273

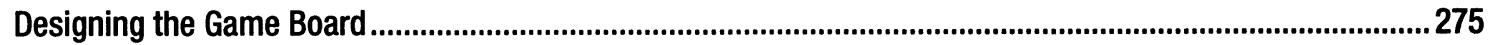

Creating the Packet Object..........................................................................................................................278

Setting Up the View Controller Header ..........................................................................................................280

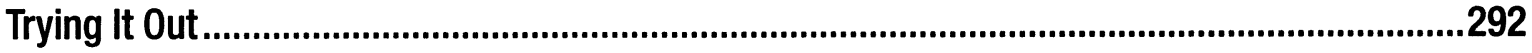

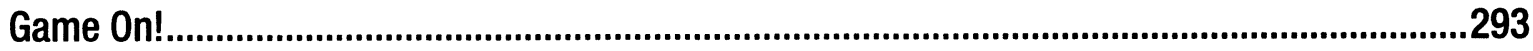

Chapter 10: Map Kit..........................................................................................295

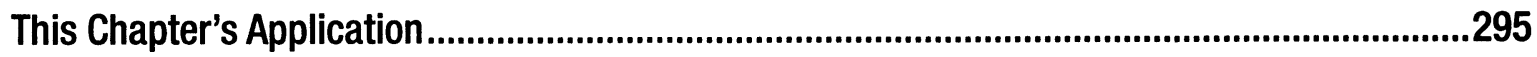

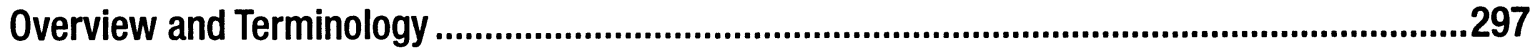

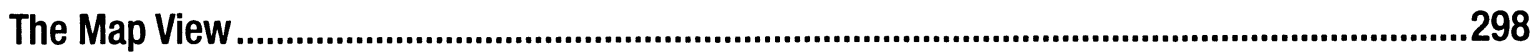

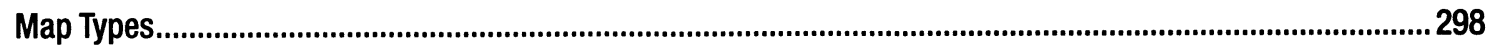

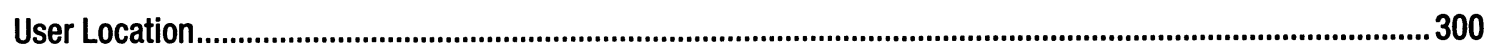

Coordinate Regions ................................................................................................................................. 301

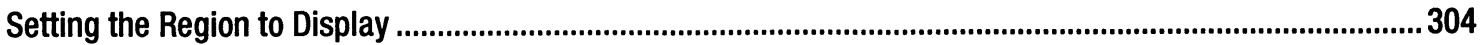

The Map View Delegate ..................................................................................................................................... 304 
Annotations .306

The Annotation Object 306

The Annotation View 307

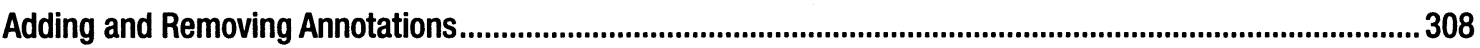

Selecting Annotations............................................................................................................................... 308

Providing the Map View with Annotation Views.............................................................................................309

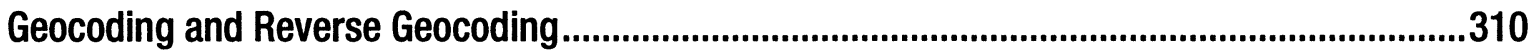

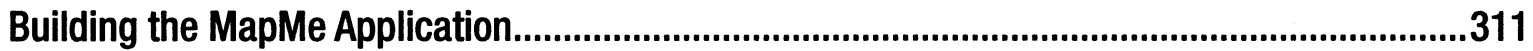

Linking the Map Kit and Core Location Frameworks......................................................................................311

Building the Interface ......................................................................................................................... 311

Finishing the View Controller Interface............................................................................................................313

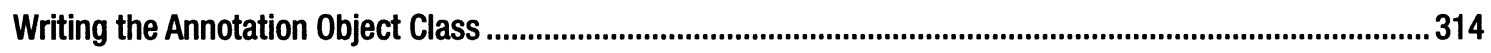

Implementing the MapMe ViewController ......................................................................................................316

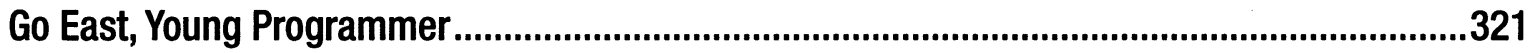

Chapter 11: Messaging: Mail, SMS, and Social Media ...............................................323

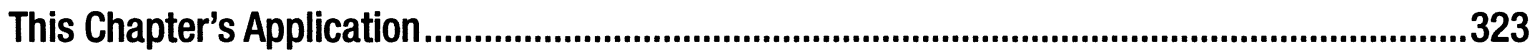

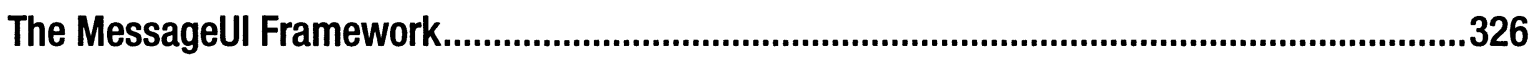

Creating the Mail Compose View Controller ..................................................................................................... 327

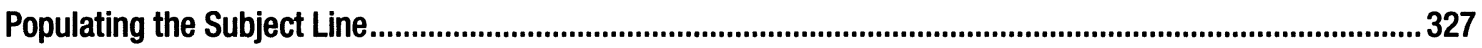

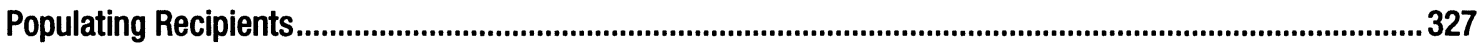

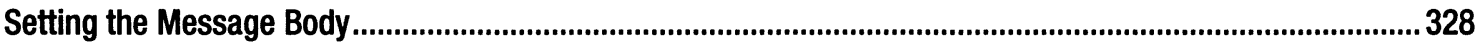

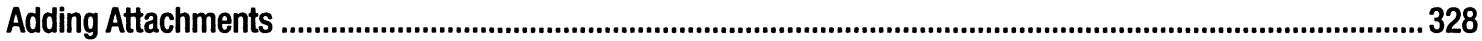

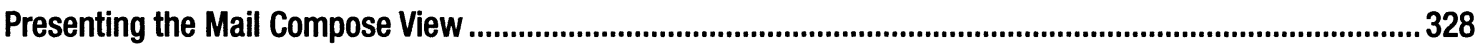

The Mail Compose View Controller Delegate Method.......................................................................................328

Message Compose View Controller .................................................................................................................... 329

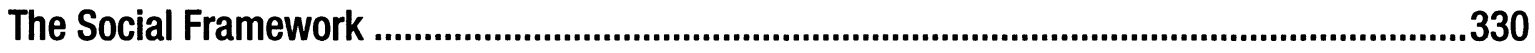

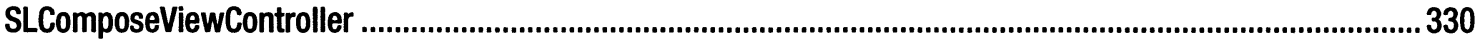

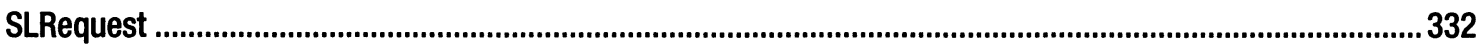

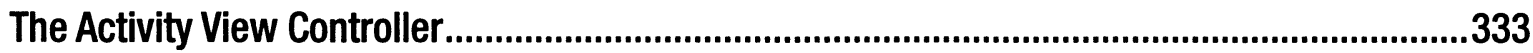


Building the Messagelmage Application

Building the User Interface 334

Taking the Picture.

Calling the Camera 334

Picking the Message Sender 335

Mailing It In...

Chapter 12: Media Library Access and Playback ................................................................337

The MediaPlayer Framework.

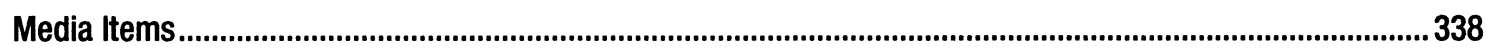

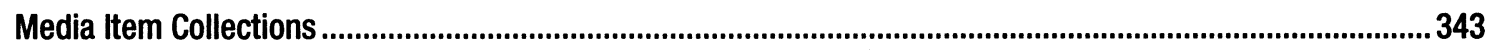

Media Queries and Media Property Predicates ...............................................................................................344

The Media Picker Controller ....................................................................................................................347

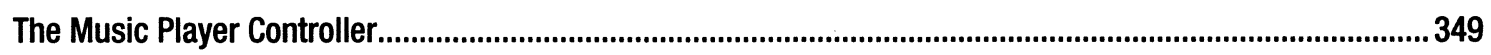

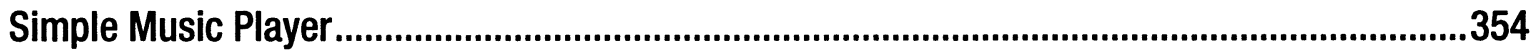

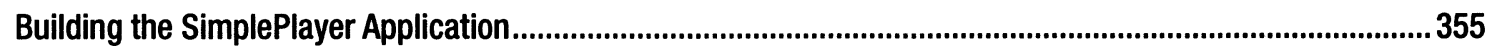

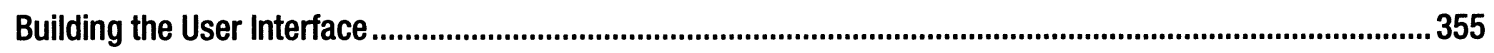

Declaring Outlets and Actions ......................................................................................................................360

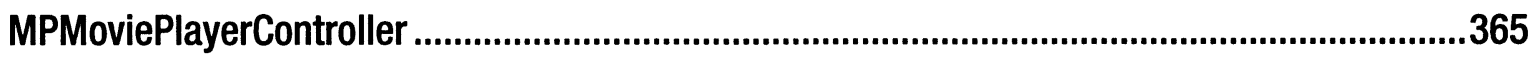

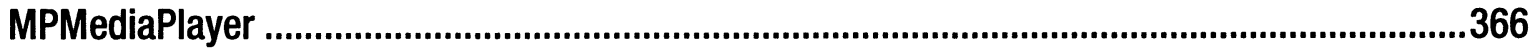

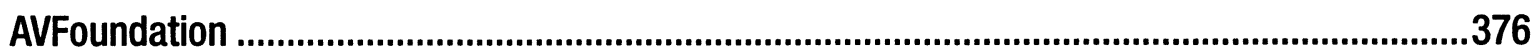

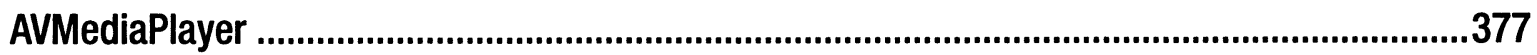

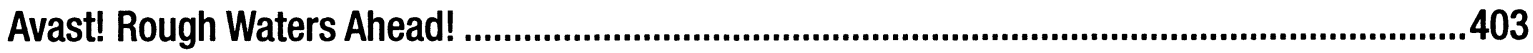

Chapter 13: Locking It Down: iOS Security...............................................................405

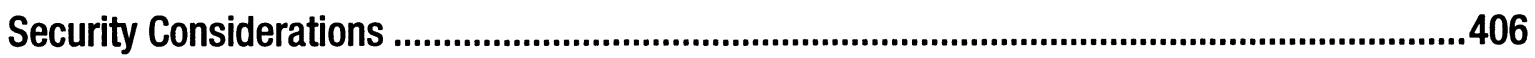

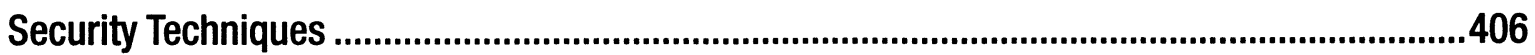

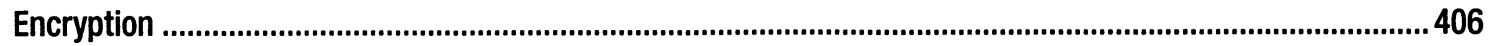

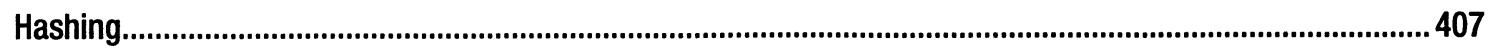

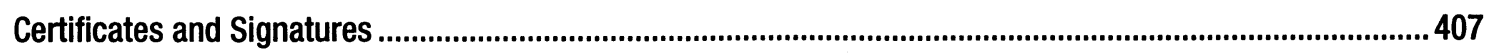

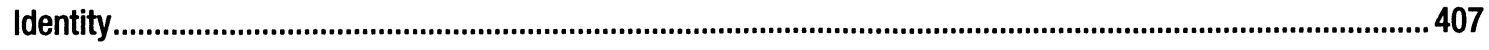


Security in iOS 407

Randomization Keychains 409

Certificates, Keys, and Trust Services.

Keychain Viewer Application

Create a Certificate Authority.

Creating the Keychain App

Security Never Sleeps

Chapter 14: Keeping Your Interface Responsive

Designing the Interface .445

Implementing the Stalled View Controller 446

Timers 449

Creating a Timer 449

Stopping a Timer

Limitations of Timers. 450

Fixing Stalled with a Timer.

Creating the Batch Object.

Updating the Nib.

Updating the View Controller Header

Updating the View Controller Implementation

Operation Queues and Concurrency.

Threads.

Operations

Operation Queues.

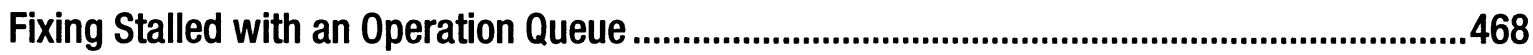

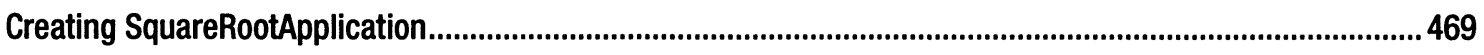

Custom ProgressCell . 
Adjusting the User Interface

Changes to ViewController.h 474

Updating ViewController.m 475

Queue 'em Up 480

\section{Chapter 15: Unit Testing, Debugging, and Instruments} 481

Unit Tests

Debugging. 491

Breakpoints .493

The Debug Navigator. .495

The Debug Area 496

Trying Out the Debug Controls. 498

The Breakpoint Navigator and Symbolic Breakpoints 500

Conditional Breakpoints .501

Breakpoint Actions. 503

Static Analysis .506

One More Thing About Debugging 507

Profiling With Instruments 507

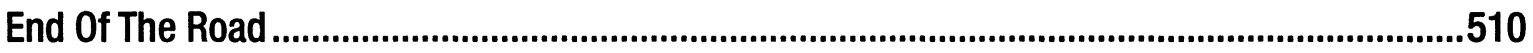

Chapter 16: The Road Goes Ever On... ................................................................................511

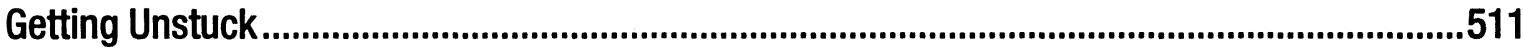

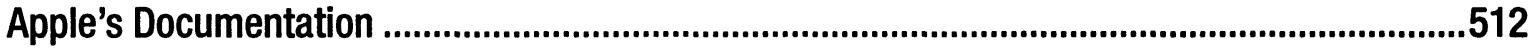

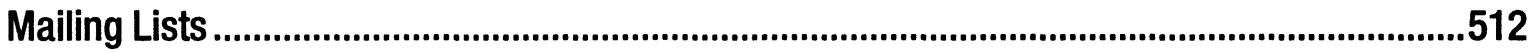

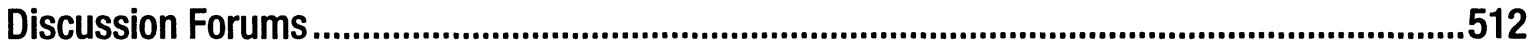

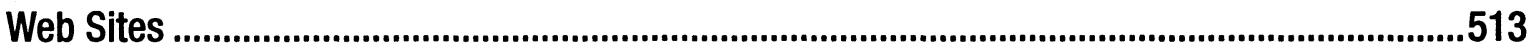

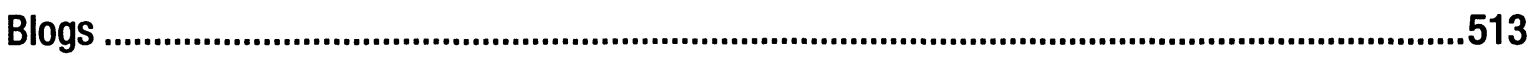

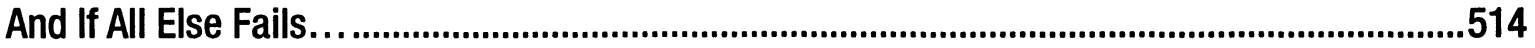

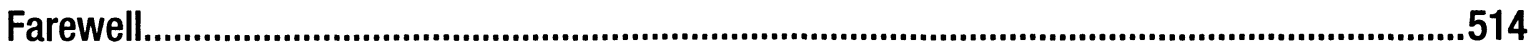




\section{About the Authors}

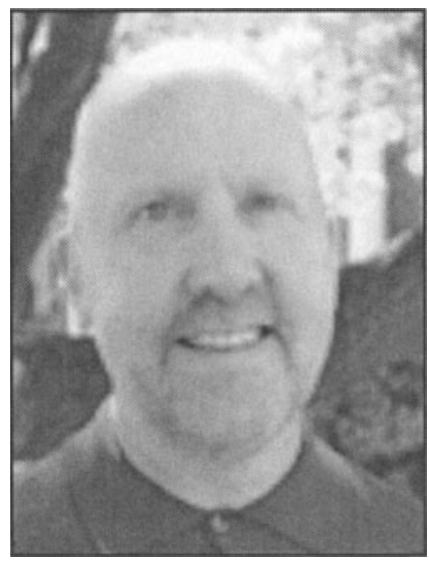

Dave Mark is a longtime Mac developer and author who has written a number of books on Mac and iOS development, including Beginning iPhone 4 Development (Apress, 2011), More iPhone 3 Development (Apress, 2010), Learn C on the Mac (Apress, 2008), Ultimate Mac Programming (Wiley, 1995), and The Macintosh Programming Primer series (Addison-Wesley, 1992). Dave was one of the founders of MartianCraft, an iOS and Android development house. Dave loves the water and spends as much time as possible on it, in it, or near it. He lives with his wife and three children in Virginia.

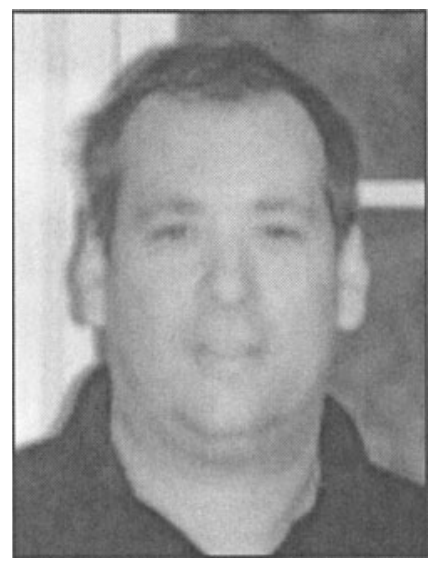

Jeff LaMarche is a Mac and iOS developer with more than 20 years of programming experience. Jeff has written a number of iOS and Mac development books, including Beginning iPhone 4 Development (Apress, 2011), More iPhone 3 Development (Apress, 2010), and Learn Cocoa on the Mac (Apress, 2010). Jeff is a principal at MartianCraft, an iOS and Android development house. He has written about Cocoa and Objective-C for MacTech Magazine, as well as articles for Apple's developer web site. Jeff also writes about iOS development for his widely read blog at www.iphonedevelopment.blogspot.com. 


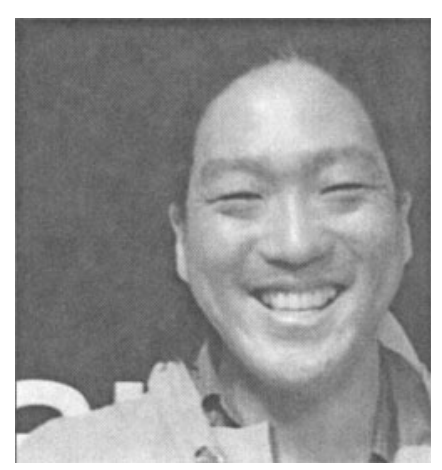

Kevin Kim is a co-founder and developer at AppOrchard LLC, a Tipping Point Partners company focused on sustainable iOS development. A graduate of Carnegie Mellon University, he was first exposed to the NeXTStep computer (the ancestor of today's iPhone) as a programmer at the Pittsburgh Supercomputing Center and has been hooked ever since. His career has spanned finance, government, biotech, and technology, including Apple where he managed the Apple Enterprise Services team for the New York metro area. Kevin was also a co-author of Pro iOS 5 Tools (Apress, 2011). He currently resides in the Alphabet City section of New York City with his wife and a clowder of rescued cats. 


\section{About the Technical Reviewer}

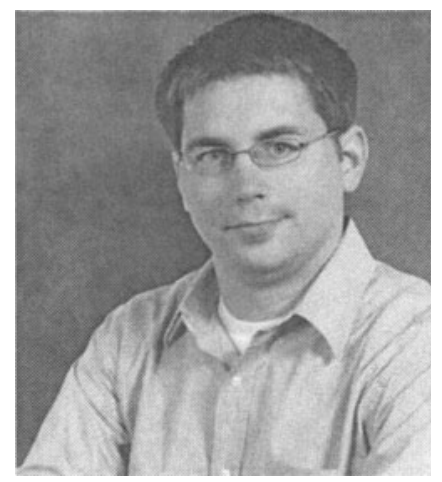

Nick Waynik has been working in the IT field for over 13 years and has done everything from network administration to web development. He started writing iOS apps when the SDK was first released. Since then he has gone on to start his own business focusing on iOS development. He loves spending his free time with his wife, Allison, and son, Preston; sometimes he even plays golf. He blogs at nickwaynik.com and can be found on Twitter as @n_dubbs. 


\section{Acknowledgments}

Writing a book like this one is more than the effort of us, the authors. Even though our names are on the cover, it is the result of the hard work of many people.

First, I would like to thank Dave Mark and Jeff LaMarche for writing the first version of the book, and for giving me a solid foundation from which to build and expand upon.

I would like to thank the staff at Apress for making sure this book was completed as close to schedule as possible. Brigid Duffy provided the guidance and oversight to make sure I completed this book. Tom Welsh made sure I stayed on topic and kept things clear. Mary Behr made the manuscript look beautiful. l'd also like to thank Brandon Levesque for making sure people knew this book was coming.

Thanks to the technical reviewers Nick Waynick and Mark Dalrymple for making sure the code I wrote actually works. Any mistakes that still exist are mine.

Thanks to my friends and colleagues at AppOrchard for their patience through the last several months of curmudgeonly behavior and for helping me make this project successful.

A great deal of thanks to my wife, Annie, for making sure I worked on this book when I would have rather been watching baseball or playing guitar. Thanks to my cats, PK, Manny, and Leela, for wanting to be fed when I needed a break. An extra thanks goes to Manny for being the subject of many of the examples in this book.

Finally, thanks to you, the reader, for buying this book. We like to think of programming as a scientific discipline, but, at times it feels more like a black art. If this book helps you on your journey of understanding iOS programming, then it is all worthwhile. 